\title{
Optimization of large amorphous silicon and silica structures for molecular dynamics simulations of energetic impacts
}

\section{Citation}

Samela, Juha, Scott A. Norris, Kai Nordlund, and Michael J. Aziz. 2011. “Optimization of Large Amorphous Silicon and Silica Structures for Molecular Dynamics Simulations of Energetic Impacts." Nuclear Instruments and Methods in Physics Research Section B: Beam Interactions with Materials and Atoms 269 (14) (July): 1568-1571. doi:10.1016/j.nimb.2010.11.017.

\section{Published Version}

doi:10.1016/j.nimb.2010.11.017

\section{Permanent link}

http://nrs.harvard.edu/urn-3:HUL.InstRepos:34264571

\section{Terms of Use}

This article was downloaded from Harvard University's DASH repository, and is made available under the terms and conditions applicable to Open Access Policy Articles, as set forth at http:// nrs.harvard.edu/urn-3:HUL.InstRepos:dash.current.terms-of-use\#OAP

\section{Share Your Story}

The Harvard community has made this article openly available.

Please share how this access benefits you. Submit a story.

Accessibility 


\title{
Optimization of large amorphous silicon and silica structures for molecular dynamics simulations of energetic impacts
}

\author{
Juha Samela ${ }^{a, *}$, Scott A. Norris ${ }^{1}$, Kai Nordlund ${ }^{a}$, Michael J. Aziz ${ }^{c}$ \\ ${ }^{a}$ Department of Physics and Helsinki Institute of Physics, University of Helsinki, P.O. \\ Box 43, FI-00014 University of Helsinki, Finland \\ ${ }^{b}$ Southern Methodist University, Dallas, TX 75205, USA \\ ${ }^{c}$ School of Engineering and Applied Sciences, Harvard University, 29 Oxford St., \\ Cambridge, MA 02138, USA
}

\begin{abstract}
A practical method to create optimized amorphous silicon and silica structures for molecular dynamics simulations is developed and tested. The method is based on the Wooten, Winer, and Weaire algorithm and combination of small optimized blocks to larger structures. The method makes possible to perform simulations of either very large cluster hypervelocity impacts on amorphous targets or small displacements induced by low energy ion impacts in silicon.
\end{abstract}

Keywords:

Molecular-dynamics simulation, Silicon, Silica, Amorphous network 


\section{Introduction}

Quality of amorphous structures used in molecular dynamics (MD) simulations is a critical factor to reliable results in many applications. For example, nanopattern formation on silicon surfaces under ion beam irradiation is a coherent effect of small displacements of atoms induced by individual impacts [1]. To detect these displacements, the amorphous structure should be free of internal stresses and its density should correspond to the density of the real material. On the other hand, billion atom simulations of cluster impacts require targets whose density and mechanical properties correspond real materials [2]. The aim is to create minimally strained continous random networks of these materials.

The Wooten, Winer, and Weaire (WWW) [3] algorithm is considered one of the best optimization methods to create high-quality amorphous structures for simulations and better than generation of structures with $\operatorname{MD}[4,5,6]$, although some criticism has been presented [5]. However, it is not possible to fully optimize structures that are large enough for many MD applications like impact simulations. Structures of only a few hundred thousand atoms can be optimized [5].

Based on the WWW algorithm, We have developed a practical method to create amorphous silicon and silica structures that give satisfactory results in impact simulations. First, we apply the WWW algorithm to optimize several nanometer wide amorphous structures. Then we use these small structures as building blocks of larger structures. Finally, the structure is further annealed and relaxed using the particular interatomic potential applied in the impact simulations. The quality of modelled structures are then compared to 
experimental data. However, there are differences in published experimental reference data because the real structures of amorphous materials depend on the method used to produce them. Therefore, it is necessary to first decide what kind of modelled amorphous structure is wanted. In this work, the quality criteria for the modelled structures are random location of atoms, rather high average density, and absence of large voids. These particular criteria are chosen to get structures which can be used to detect small displacements of atoms induced by ion bombardment.

\section{Methods}

The WWW implementation used in this study is described in Ref. [6]. It is implemented for parallel operation and it is portable to common parallel computing systems. In this study, it was run on a Cray XT4/XT5 system where the optimization of a $10 \mathrm{~nm}$ wide a-Si block takes about three days using 16 CPUs. During the WWW optimization, a Keating potential was used for both a-Si and a-SiO ${ }_{2}[7,6]$. The energy minimization is done locally in order to get $O(N)$ scaling [6].

The MD simulation arrangements are described in Refs. $[8,9,10]$. The environment-dependent interatomic potential (EDIP) [11] and the StillingerWeber (SW) potential [12] were used for a-Si. For silica, the Watanabe potential was used in the simulations $[13,14,15]$.

Rectangular blocks of a-Si and a-SiO2 were optimized in two phases starting from random atomic configurations. First the block was processed using the WWW method until the total potential energy changes become negligible, which does not mean that the system has reached the global potential 
energy minimum. For the big blocks, it was necessary to slightly modify the optimization parameters during the last phases of the optimization to remove the system from too high local energy minimum. For example, some blocks were temporarily allowed to expand which gives more freedom to atoms to move to lower energy positions. In the second phase, the blocks were annealed in MD to $1 \mathrm{~K}$ using periodic boundary conditions and a more advanced interaction model, like the EDIP or SW potentials. The ambient temperature $1 \mathrm{~K}$ was used because the structures will be used to verify a theoretical rippling model in the case where thermal effects are not present [1].

Because periodic boundary conditions were used, identical copies of a block can be put side by side to form a larger structure. These combined structures were annealed to $1 \mathrm{~K}$ with MD to relax possible stresses. The result is an optimized amorphous structure which has a medium-range periodicity due to the block structure. Finally, one face of the structure is opened for the ion bombardment and the system is annealed again with MD.

\section{Results}

With the WWW method, it is usually not possible to optimize arbitrary large amorphous structures. Increasing the number of CPUs does not compensate the effect that the optimization of larger structures practically stops near a local energy minimum. The reason to this is the following: The barriers in the energy landscape around the local minima becomes larger in average when the block size increases. Sooner or later, the probability that a practically possible series of WWW optimization steps would raise the system over the barrier becomes very small. In tiny systems, like a $3 \times 3 \times 3$ 
nm a-Si block, this happens almost at the global minimum, resulting in a very well optimized structure. When the block size increases, the energy minimum reached is considerably higher than the global minimum. At the moment, the practical limit for the block side is around $10 \mathrm{~nm}$ according to test simulations made in this study with a few hundred thousand atoms. If the quality of the four-fold coordination of $\mathrm{Si}$ atoms is critical from the applications point of view, the limit is even lower.

In the $3 \times 3 \times 3 \mathrm{~nm}$ EDIP a-Si block $99.4 \%$ of atoms have coordination number 4. Fig. 1 shows that the structure is well-optimized compared to experimental pair correlation, bond length distribution and bond-angle distribution. However, the bond lengths become realistic only after the EDIP annealing. The SW structure based on the same WWW optimized block is almost as good. $96 \%$ of atoms have coordination number 4 .

The $10 \times 10 \times 10 \mathrm{~nm}$ a-Si block represents the upper limit of the structures that are possible to be optimized. A WWW optimized block was annealed with both the EDIP and the SW potentials. The block contains about 220,000 atoms and now only $62 \%$ (EDIP) and $66 \%$ (SW) atoms have coordination number 4. The densities are 2.49 (EDIP) and $2.41 \mathrm{~g} / \mathrm{cm}^{3}(\mathrm{SW})$. A more detailed analysis shows that a considerable portion of atoms in the EDIP block have five neighbours (Fig. 2), whereas in the SW block many atoms have less than four neighbours within the interaction distance. The EDIP silicon is very dense but in the SW silicon there are more voids where the atoms have too few neighbors. In both cases the Si-Si bonds are too long which indicates stress in the structure. It is clear that the EDIP structure is better when effects of the ion impacts are simulated because there is no 
danger that the voids collapse upon impact which would make it impossible to detect the small displacement field induced by impacts. Our test simulations confirm this. In conclusion, the potentials give very different structures, although the comparison to the experimental results shows (Fig. 3) that the both structures are equally good in average. If the structure is initially not very near the global energy minimum the MD annealing affects it depending on the potential.

A large combined EDIP a-Si structure was tested in $500 \mathrm{eV}$ Ar impact simulations. A $20 \times 20 \times 10$ combined structure was made of four $10 \times 10 \times 10$ blocks and then annealed. The analysis of displacements confirms that the structure is very stable and does not contain areas which could easily deform upon impact and create artificial effects in the atom displacement field. Fig. 4 shows the cross-section of the displacement field when no impact occurs. The field does not show any periodicity although there are four identical blocks present. The displacements seem to be due to a global relaxation, which becomes possible when identical blocks are placed side by side and the surface is opened. In spite of the partial optimization, the structure makes possible to detect yery small displacements in low-energy impact simulations.

Figs. 5 and 6 show the comparison of optimized and MD annealed a- $\mathrm{SiO}_{2}$ structures to experimental results. The structure was made of $5 \times 5 \times 2 \mathrm{~nm}$ blocks. This particular silica structure is used in large-scale cluster impact simulations where the uniform density of the material is critical [2]. The density is $2.15 \mathrm{~g} / \mathrm{cm}^{3}$, which is comparable to the experimental density 2.2 $\mathrm{g} / \mathrm{cm}^{3}$. The average O-Si-O angle is $108.6^{\circ}$ indicating that the tetrahedral structure typical to silica is achieved in the optimization. Note that the 
density and the Si-O-Si angle distribution of the real silica vary depending on the type of the material, as the experimental results in Fig. 6 show. The variation among silica structures is even larger than among a-Si structures.

\section{Discussion}

The method of building large structures of small optimized blocks provides an opportunity to simulate high energy ion or cluster impacts on amorphous materials. This is not possible with structures annealed only with MD because these structures are not usually dense enough and collapse upon impact. However, the periodicity of the structures may affect the results of the simulations. To minimize this effect, the dimensions of the building blocks should be clearly different than the size of the dynamic phenomenon which is simulated. For example, the crater formation and shock wave propagation are not affected in cluster bombardment simulation, where the block size is considerably smaller than the cavity induced by the cluster impact. On the other hand, the blocks should be much larger than the collision cascade area of a single ion impact, otherwise systematic patterns may appear in the results. For example, it was necessary to use $10 \mathrm{~nm}$ building block instead of the $3 \mathrm{~nm}$ in simulations of $500 \mathrm{eV}$ Ar impact on a-Si.

A combined structure is not just a sum of its constituents. When a small block that is annealed using periodic boundaries is put side by side with identical blocks, its environment does not change and the structure is very static. However, when a face of a combined structure is opened to form a surface for ion bombardment, the structure may change because the opening introduces more freedom to atoms at the surface to slightly change their positions. 
Eventually, this affects the whole structure. For example, a structure made of $3 \times 3 \times 3 \mathrm{~nm}$ a-Si blocks that was well optimized (Fig. 1) went through a small but global artificial displacement of most atoms during the impact simulation. These effects are negligible in high-energy cluster impact simulations, but harmful when very small atomic displacements should be detected. Instead, the structures made of the partially optimized $10 \times 10 \times 10 \mathrm{~nm} \mathrm{a-Si}$ blocks proved to be reasonably good in ion bombardment simulations in spite of the incomplete optimization (Fig. 3.

\section{Conclusions}

We have shown that the WWW method and combination of small welloptimized a-Si and a- $\mathrm{SiO}_{2}$ blocks can be used in practice to create amorphous targets for MD simulations of both large clusters and low-energy ions. The density and characteristics of these amorphous structures correspond very well to the properties of real structures. The majority of the Si atoms are four-fold coordinated. The amorphous networks are free of internal stresses which makes it possible to detect weak displacement fields induced by 100 eV Ar atom impacts, for example. However, the structures must be carefully annealed and analyzed to avoid artificial effects.

\section{Acknowledgments}

Generous grants of computer time from the Center for Scientific Computing in Espoo, Finland are gratefully acknowledged. We thank Dr. S. von Alfthan for providing us the WWW code. This work was performed 
within the Finnish Centre of Excellence in Computational Molecular Science (CMS), financed by The Academy of Finland and the University of Helsinki.

\section{References}

[1] S. A. Norris, M. P. Brenner, M. J. Aziz, From crater functions to partial differential equations: a new approach to ion bombardment induced nonequilibrium pattern formation, J. Phys.: Condens. Matter 21 (2009) 224017 (11pp).

[2] J. Samela, K. Nordlund, Classical molecular dynamics simulations of hypervelocity nanoparticle impacts on amorphous silica, Phys. Rev. B $81(2010) 054108$.

[3] F. Wooten, K. Winer, D. Weaire, Computer generation of structural models of amorphous si and ge, Phys. Rev. Lett. 54 (1985) 1392-1395.

[4] Y. Tu, J. Tersoff, G. Grinstein, D. Vanderbilt, Properties of a continuous-random-network model for amorphous systems, Phys. Rev. Lett. 81 (1998) 4899-4902.

[5] R. L. C. Vink, G. T. Barkema, Large well-relaxed models of vitreous silica, coordination numbers, and entropy, Phys. Rev. B 67 (2003) 245201.

[6] S. von Alfthan, A. Kuronen, K. Kaski, Realistic models of amorphous silica: A comparative study of different potentials, Phys. Rev. B 68 (2003) 073203. 
[7] P. N. Keating, Effect of invariance requirements on the elastic strain energy of crystals with application to the diamond structure, Phys. Rev. 145 (1966) 637-645.

[8] G. Kornich, G. Betz, V. Zaporojtchenko, K. Pugina, Low energy ion bombardment of metal nanoclusters on graphite, Nucl. Instr. Meth. Phys. Res. B 255 (2007) 233-237.

[9] M. Ghaly, K. Nordlund, R. S. Averback, Molecular dynamics investigations of surface damage produced by kiloelectronvolt self-bombardment of solids, Phil. Mag. A 79 (1999) 795-820.

[10] K. Nordlund, M. Ghaly, R. S. Averback, M. Caturla, T. D. de la Rubia, J. Tarus, Defect production in collision cascades in elemental semiconductors and fcc metals, Phys. Rev. B 57 (1998) 7556-7570.

[11] J. F. Justo, M. Z. Bazant, E. Kaxiras, V. V. Bulatov, S. Yip, Interatomic potential for silicon defects and disordered phases, Phys. Rev. B 58 (1998) 2539-2550.

[12] F. H. Stillinger, T. A. Weber, Computer simulation of local order in condensed phases of silicon, Phys. Rev. B 31 (1985) 5262-5271.

[13] H. Ohta, S. Hamaguchi, Classical interatomic potentials for Si-O-F and Si-O-Cl systems, J. Chem. Phys. 115 (2001) 6679-6690.

[14] T. Watanabe, D. Yamasaki, K. Tatsumura, I. Ohdomari, Improved interatomic potential for stressed si, o mixed systems, Appl. Surf. Sci. 234 (2004) 207-213. 
[15] J. Samela, K. Nordlund, V. N. Popok, E. E. B. Campbell, Origin of complex impact craters on native oxide coated silicon surfaces, Phys. Rev. B 77 (2008) 075309.

[16] L. Pusztai, S. Kugler, Comparison of the structures of evaporated and ion-implanted amorphous silicon samples, J. Phys.: Condens. Matter 17 (2005) 2617-2624.

[17] S. Kugler, Z. Várallyay, Possible unusual atomic arrangements in the structure of amorphous silicon, Phil. Mag. Lett. 81 (2001) 569-574.

[18] D. I. Grimley, A. C. Wrighta, R. N. Sinclair, Neutron scattering from vitreous silica iv. time-of-flight diffraction, J. Non-Cryst. Solids 119 (1990) 49-64.

[19] X. Yuan, A. N. Cormack, Si-o-si bond angle and torsion angle distribution in vitreous silica and sodium silicate glasses, J. Non-Cryst. Solids 319 (2003) 31-43. 

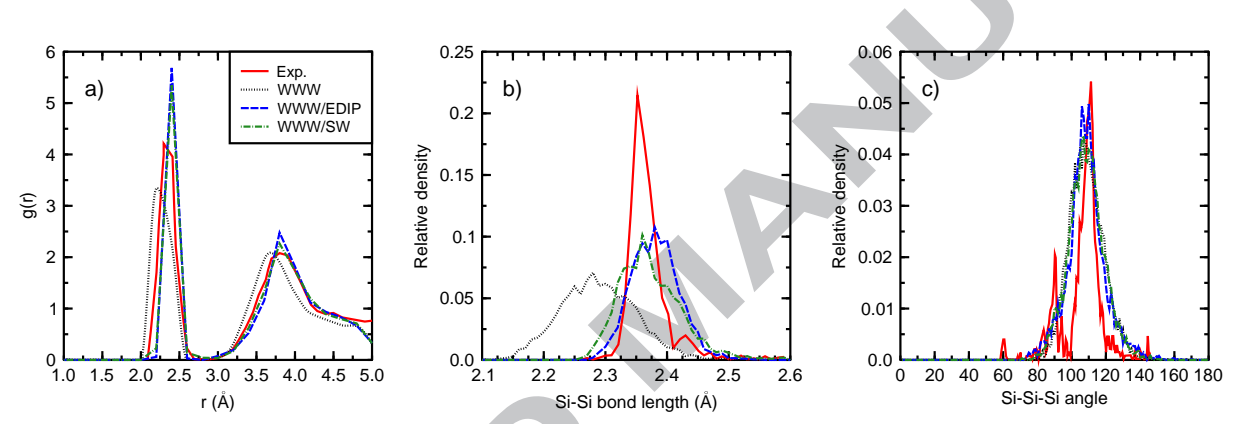

Figure 1: (Color online) Pair correlation (a), Si-Si bond length distribution (b), and Si-SiSi angle distribution (c) of the $3 \times 3 \times 3$ block the EDIP and SW annealing compared to the corresponding experimental results. The intermediate result after the WWW optimization is also shown. The experimental pair correlation is from Ref. [16]. The bond length and agle distributions are from Ref. [17]. 


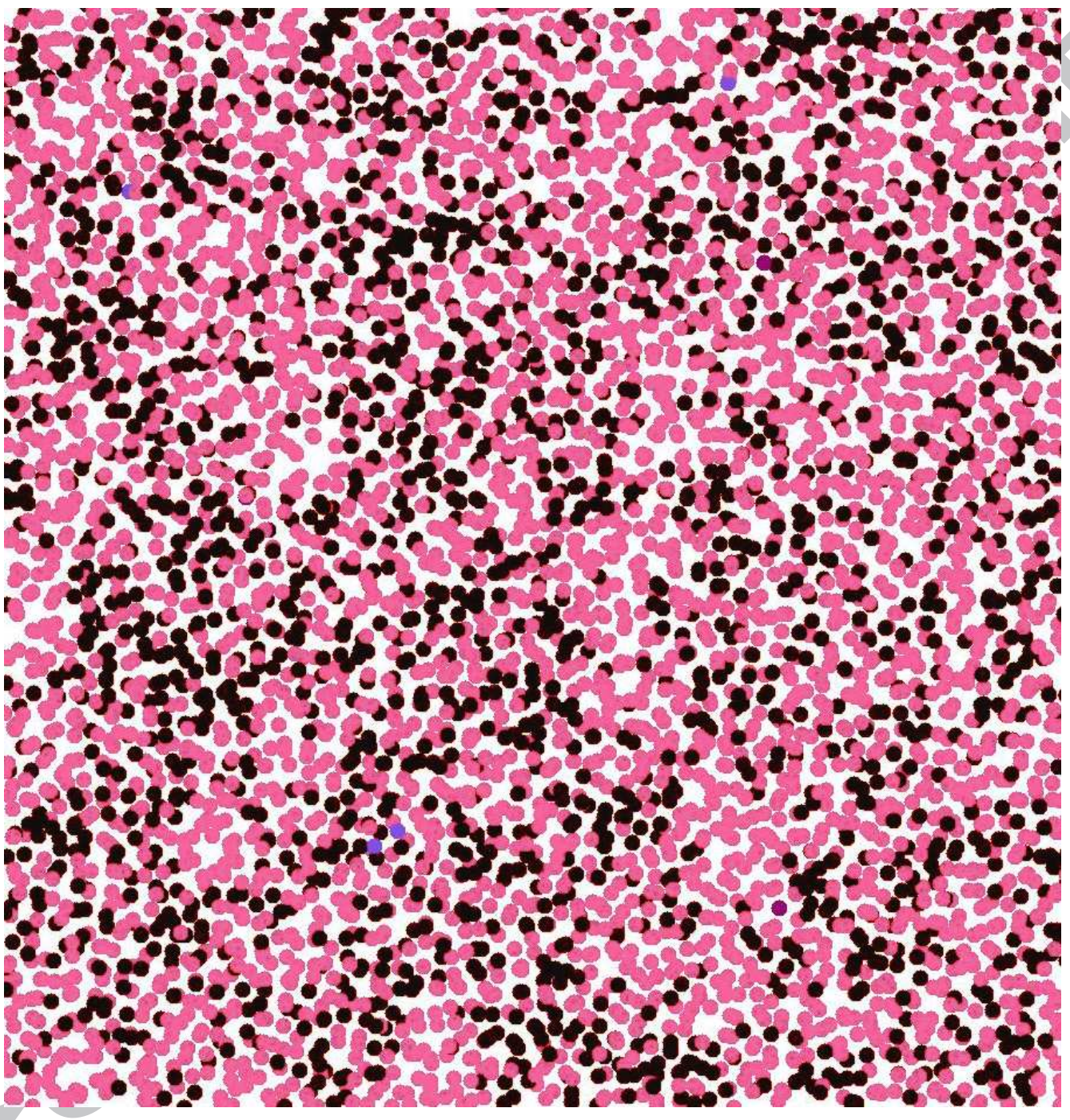

Figure 2: (Color online). A cross-section of the optimized $10 \times 10 \times 10$ a-Si structure (EDIP). Atoms that have five neighbors are marked with a darker color. These coordination defects are randomly distributed over the volume. The structure has no large voids. 

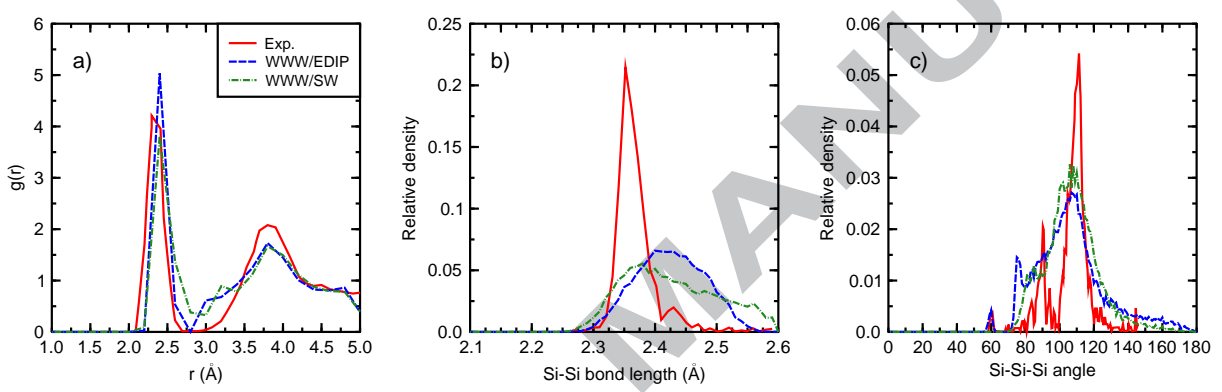

Figure 3: (Color online) Pair correlation (a), Si-Si bond length distribution (b), and Si-Si-Si angle distribution of the $10 \times 10 \times 10$ block after the EDIP and SW annealing compared to the corresponding experimental results. The experimental pair correlation is from Ref. [16]. The bond length and angle distributions are from Ref. [17]. Note that comparisons of a-Si structure parameters are always approximate because the structure of a-Si depends on the method used to produce it. 


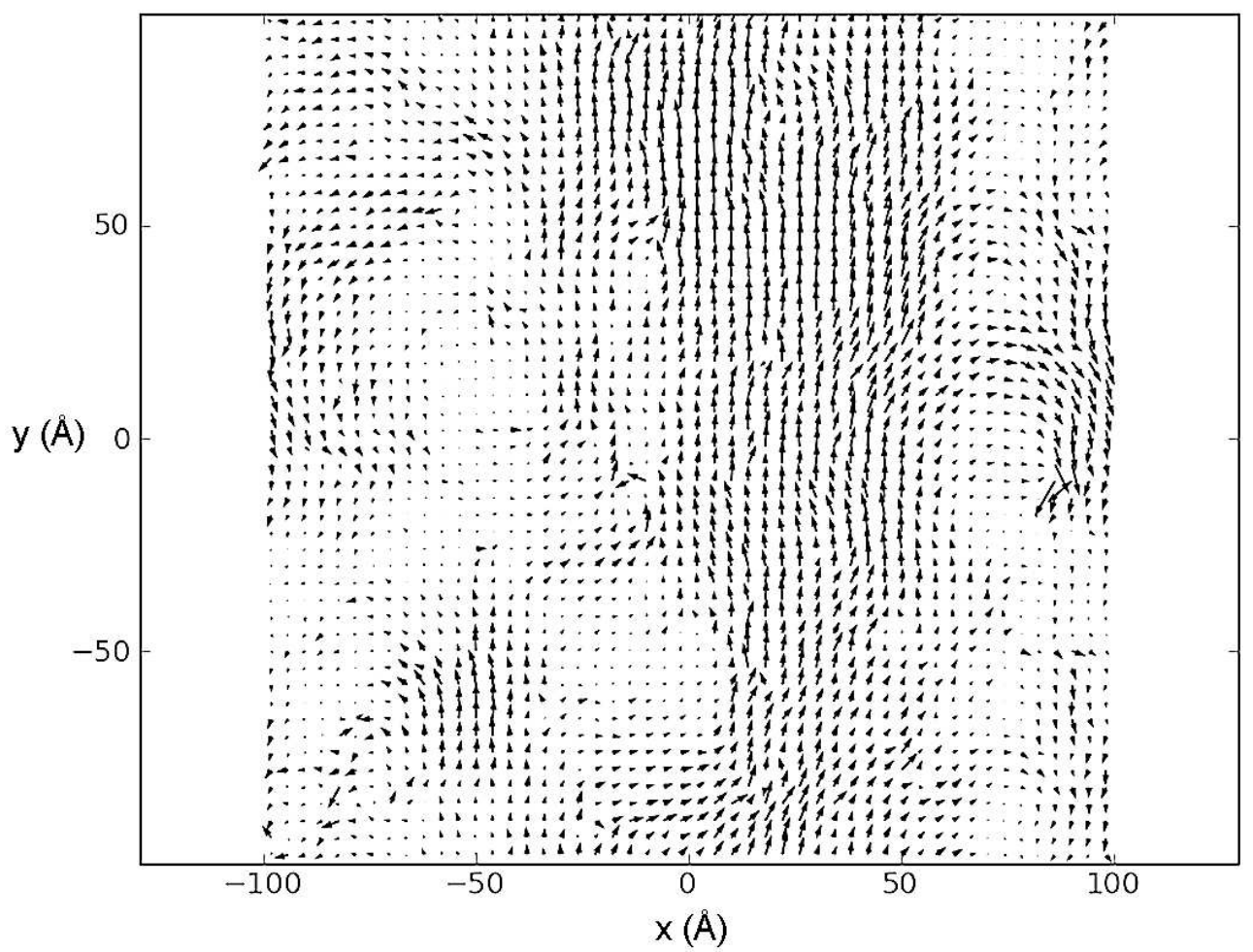

Figure 4: (Color online) Cross section of the atom displacement field at the surface of the combined a-Si block. The displacements are calculated after a 25 ps MD simulation by comparing the final positions to the initial positions of the atoms. Most of the atomic displacements shown as small arrows are less than $0.5 \AA$. 


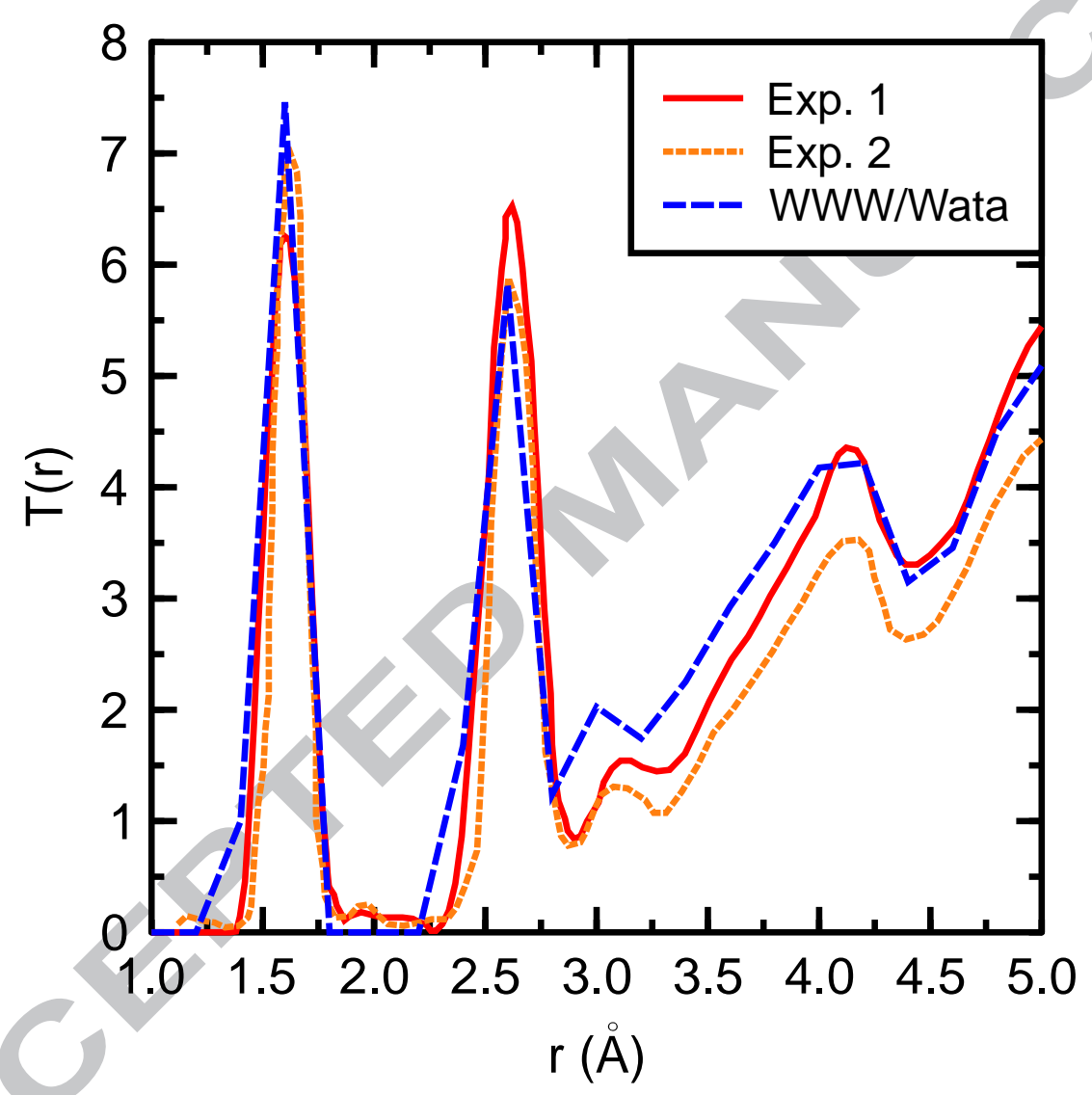

Figure 5: (Color online) Pair correlation of the $\mathrm{SiO}_{2}$ block after relaxation with the Watanabe potential compared to experimental results: Exp. $1[6,18]$ and Exp. $2[6,19]$. 


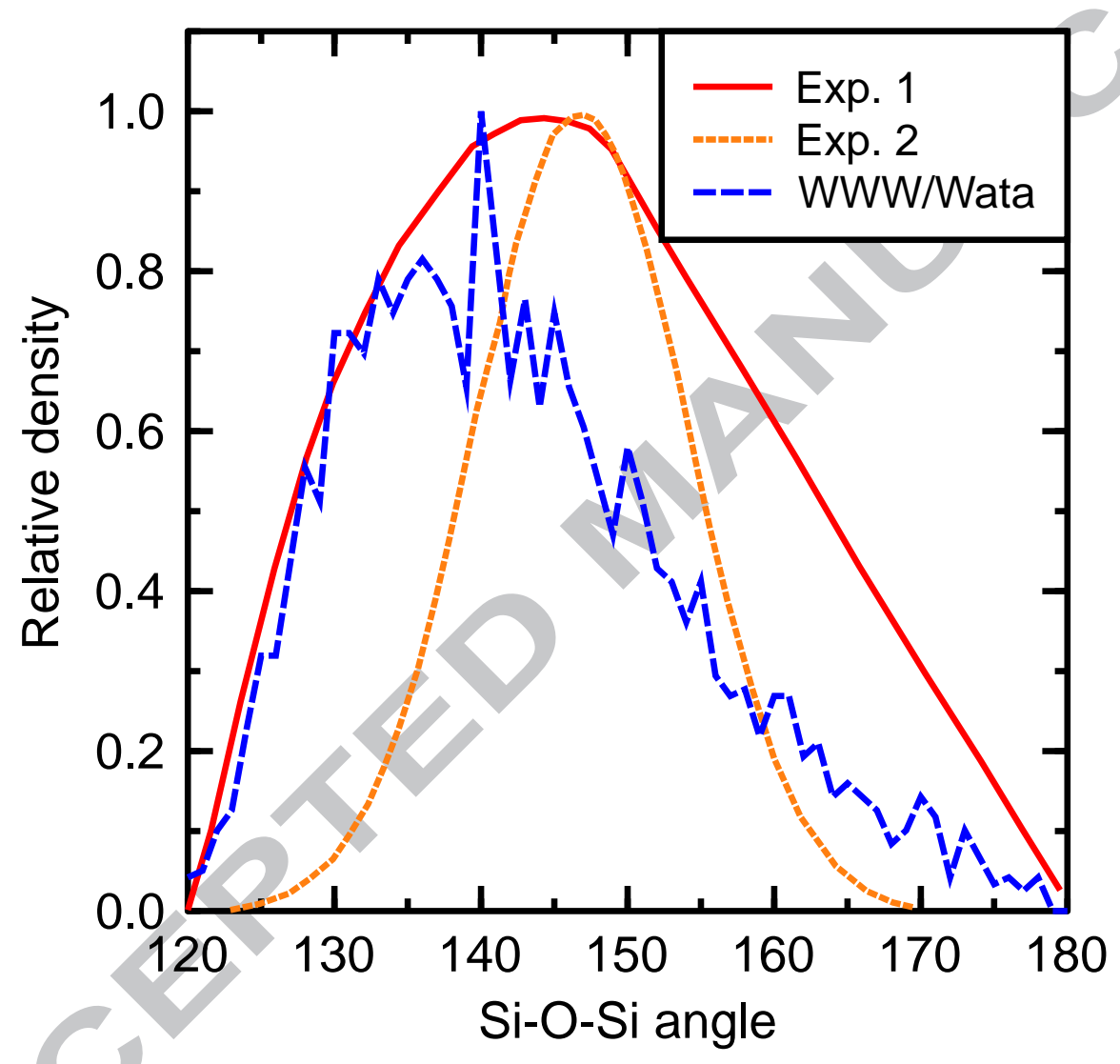

Figure 6: (Color online) Si-O-Si angle distribution of the $\mathrm{SiO}_{2}$ block after relaxation with the Watanabe potential compared to experimental results: Exp. $1[6,18]$ and Exp. $2[6,19]$. 